\title{
Efficacy, safety and pharmacokinetics of bosentan in portopulmonary hypertension
}

\author{
Laurent Savale*,\#,ף,f, Romain Magnier ${ }^{+, f}$, Jérôme Le Pavec*,\#, Xavier Jaïs*,\#,ף, \\ David Montani*,\#, , Dermot S. O'Callaghan*,\#,ף, Marc Humbert*,\#, , \\ Jasper Dingemanse ${ }^{\S}$, Gérald Simonneau*,\#, and Olivier Sitbon*,\#,ף
}

ABSTRACT: Data on treatment of patients with portopulmonary hypertension (PoPH) are limited, as they are usually excluded from randomised controlled trials with pulmonary arterial hypertension (PAH)-specific therapies. This study investigated the short- and long-term efficacy/safety of bosentan in these patients, as well as its pharmacokinetics.

All 34 consecutive patients with PoPH treated with first-line bosentan (December 2002 to July 2009) were retrospectively evaluated. Assessments included the New York Heart Association functional class (NYHA FC), blood tests, haemodynamics, 6-min walk distance (6MWD) and eventfree status. The pharmacokinetics of bosentan in five patients with Child-Pugh (C-P) class B cirrhosis were compared with idiopathic PAH patients.

Significant improvements from baseline were observed in NYHA FC, 6MWD and haemodynamics, and were largely maintained during follow-up. Patients with C-P class B cirrhosis $(n=9)$ had significantly larger haemodynamic improvement after mean \pm SD $5 \pm 2$ months. Mean followup time was $43 \pm 19$ months; four patients died and seven patients had significant elevation of liver enzymes (annual rate 5.5\%). Plasma concentrations of bosentan were higher in patients with C-P class B cirrhosis than those observed in idiopathic PAH.

These data confirm the benefit of bosentan treatment for patients with PoPH. Haemodynamic improvements were particularly pronounced in patients with more severe cirrhosis. The safety profile of bosentan was consistent with previous studies.

KEYWORDS: Bosentan, cirrhosis, endothelin receptor antagonists, hypertension, portopulmonary hypertension, pulmonary

P ortopulmonary hypertension (PoPH) is defined as pulmonary arterial hypertension (PAH) associated with portal hypertension, with or without advanced hepatic disease. The disease is classified as group 1 pulmonary hypertension $(\mathrm{PH})$ in the current $\mathrm{PH}$ classification [1]. Although it shares many characteristics with idiopathic PAH (IPAH), including pathophysiological changes in lung microvasculature, the haemodynamics of $\mathrm{PoPH}$ can be somewhat different at baseline with higher cardiac output and lower pulmonary vascular resistance.

According to estimates, $2-6 \%$ of patients with portal hypertension and $\sim 1-2 \%$ of those with cirrhosis have $\mathrm{PoPH}$ [2-5]. $\mathrm{PoPH}$ is a relatively common cause of $\mathrm{PH}$, representing $15 \%$ of patients enrolled in the French PAH registry [6]. Estimates of survival in patients with $\mathrm{PoPH}$ have varied widely among published studies [7-10]. The US Registry to Evaluate Early and Long-Term PAH Disease Management (REVEAL) database, in which data from $>2,700$ patients were collected, identified $\mathrm{PoPH}$ as an independent predictor of increased mortality among PAH patients (hazard ratio 3.6, 95\% CI 2.4-5.4) [11]. In contrast, LE PAVEC et al. [7] found that 1-, 3- and 5-yr survival rates among patients followed at the French National Referral Centre (Hôpital le Kremlin Bicêtre, Bicêtre, France) were $88 \%, 75 \%$ and $68 \%$, respectively, suggesting that these patients may in fact have less severe outcomes [7]. Poorer prognosis was associated with Child-Pugh (C-P) class B or C cirrhosis, high right atrial pressure and low cardiac index. Differences in the severity of liver disease at time of first assessment among the different series may in part explain the discordance of findings.

In contrast with other types of associated $\mathrm{PAH}$, patients with $\mathrm{PoPH}$ have been excluded from the large clinical trials in $\mathrm{PAH}$ and there have been no controlled studies of PAH-specific therapies exclusively in PoPH. The majority of data on
AFFILIATIONS

*Université Paris-Sud 11, Faculté de Médecine,

${ }^{\#}$ AP-HP, Centre de Référence de I'Hypertension Pulmonaire Sévère, Hôpital Bicêtre, Le Kremlin Bicêtre, "INSERM U999 "Hypertension Artérielle Pulmonaire,

Physiopathologie et Innovation Thérapeutique", Centre Chirurgical Marie-Lannelongue, Le PlessisRobinson, and

${ }^{+}$Service de Pneumologie, Centre Hospitalier Universitaire, Caen, France.

${ }^{\S}$ Actelion Pharmaceuticals Ltd, Dept of Clinical Pharmacology, Allschwil, Switzerland.

${ }^{f}$ These authors contributed equally to this article.

CORRESPONDENCE

0 . Sitbon

Service de Pneumologie

Hôpital Bicêtre

78 rue du général Leclerc 94270 Le Kremlin Bicêtre

France

E-mail: olivier.sitbon@bct.aphp.fr

Received:

July 102011

Accepted after revision:

May 142012

First published online:

May 312012

European Respiratory Journa

Print ISSN 0903-1936

Online ISSN 1399-3003 
treatment of $\mathrm{PoPH}$, therefore, derive from case series and case reports. A number of such reports suggest that the dual endothelin receptor antagonist (ERA) bosentan is well tolerated and effective in this population [12-18]. In a small study, treatment with bosentan proved to be efficacious and well tolerated in patients with C-P class A cirrhosis [19, 20]. Furthermore, the pharmacokinetics of bosentan and its metabolites in patients with C-P class A did not differ to a relevant extent from those in healthy subjects [21]. However, to date, there has been only one case report of treatment with bosentan in a single patient with more advanced liver disease [17]. This lack of data is mainly due to safety concerns associated with impaired metabolism and potentially increased risk of liver abnormalities in patients with advanced liver diseases. Indeed, bosentan is not approved for C-P class B and C patients.

This present study provides data on both the short- and longterm efficacy of the use of bosentan in patients with $\mathrm{PoPH}$, as well as safety, tolerability and some pharmacokinetic data. It is the first study to investigate the effect of treatment in patients with PoPH and cirrhosis, which included C-P class B patients.

\section{PATIENTS AND METHODS \\ Study subjects}

This was a retrospective study that aimed to describe the shortand long-term outcome of unselected patients with $\mathrm{PoPH}$ treated with first-line bosentan, including patients with noncirrhotic portal hypertension and patients with C-P class $\mathrm{A}$ and $\mathrm{B}$ cirrhosis.

Data were analysed from a total of 34 consecutive patients with PoPH treated with first-line bosentan between December 2002 and July 2009 at the French Reference Center for severe PH.

$\mathrm{PAH}$ was diagnosed by means of right heart catheterisation (RHC) showing a mean pulmonary arterial pressure $(P$ pa $)$ at rest of $>25 \mathrm{mmHg}$, a pulmonary capillary wedge pressure $<15 \mathrm{mmHg}$, and a pulmonary vascular resistance (PVR) of $>3$ Wood units [22]. Acute vasodilator testing with inhaled nitric oxide $(10 \mathrm{ppm})$ was performed in all patients, as previously described [23]. The presence of primary lung disease and post-embolic $\mathrm{PH}$ was excluded by performing pulmonary function tests, computed tomography of the chest and ventilation/perfusion lung scanning.

The diagnosis of portal hypertension was based on haemodynamic measurement of a hepatic venous pressure gradient of $>5 \mathrm{mmHg}$, or the presence of oesophageal varices at endoscopy or portal vein thrombosis diagnosed by Doppler abdominal ultrasound. Cirrhosis was documented by history of liver biopsy findings or typical clinical and/or biological signs.

According to French legislation, ethics committee agreement and provision of informed consent are not required for retrospective collection of data corresponding to current practice. The database was, however, compiled anonymously within the restrictive requirements of the Commission Nationale Informatique et Liberté, the organisation dedicated to privacy, information technology, and civil rights in France. The present study was approved by the local institutional review board (CHU Bicêtre, France). Informed consent was obtained for the pharmacokinetic study. Five patients with C-P class B cirrhosis and three patients with IPAH were included in the pharmacokinetic substudy (AC-052-114), which was approved by the ethics committee.

\section{Treatment}

All patients received nonspecific supportive therapies in accordance with current guidelines, diuretics to control signs and symptoms of right heart failure (including peripheral oedema), and long-term oxygen therapy if hypoxaemia was present [24]. Anticoagulants are not usually administered in the presence of severe hepatocellular insufficiency and/or thrombocytaemia due to hypersplenism.

Bosentan was prescribed according to the European Summary of Product Characteristics at $62.5 \mathrm{mg}$ b.i.d. for 4 weeks, followed by $125 \mathrm{mg}$ b.i.d. thereafter. All patients had liver enzyme concentrations less than three-fold the upper limit of normal (ULN) before initiation of bosentan. For seven patients (four with C-P class B cirrhosis and three with C-P class A cirrhosis), the dosing regimen was maintained at $62.5 \mathrm{mg}$ b.i.d. because of mildly increased liver enzyme concentrations less than three-fold the ULN at baseline. Liver function tests were performed every 2 weeks during the first 6 weeks and monthly thereafter. In the event of elevated liver enzymes, bosentan was stopped or the dosage was reduced, in accordance with current recommendations [25].

\section{Assessments}

All patients underwent a complete baseline evaluation before starting bosentan therapy, including assessment of modified New York Heart Association functional class (NYHA FC), physical examination, routine blood tests, non-encouraged 6min walk distance (6MWD) and resting haemodynamic variables measured by RHC. Patients were reassessed for all parameters at short- and long-term time-points. Haemodynamic assessment was repeated 3-6 months after bosentan initiation, then every year or in case of clinical worsening. Noninvasive assessments were also repeated every 4-6 months. The first and last evaluation on bosentan monotherapy were analysed for the study.

Event-free status was defined as the survival time without introduction of prostacyclin analogues and/or phosphodiesterase type- 5 inhibitor therapy, discontinuation of bosentan or acute right heart failure requiring hospitalisation for intravenous diuretics and/or dobutamine infusion.

\section{Pharmacokinetics}

Five patients with C-P class B cirrhosis were included in a pharmacokinetic substudy and compared with three patients with IPAH. Blood samples for the determination of plasma concentrations of bosentan and its three metabolites (Ro 47-8634, Ro 48-5033 and Ro 64-1056) were taken at regular intervals (predose, and 1, 2, 3, 4, 5, 6, 9 and $12 \mathrm{~h}$ after drug intake) over a dosing interval after patients had been on stable bosentan treatment for at least $\geqslant 14$ days. Ro $48-5033$ is the only pharmacologically active metabolite. Plasma concentrations were determined with a validated liquid chromatographytandem mass spectrometry assay [26]. The pharmacokinetic variables were assessed by noncompartmental analysis (WinNonlin; Pharsight, Mountain View, CA, USA). 
All patients with C-P class B cirrhosis were included in the pharmacokinetic substudy 14 days after the start of treatment with bosentan at $62.5 \mathrm{mg}$ b.i.d. Two patients with IPAH were treated with bosentan at $125 \mathrm{mg}$ b.i.d. and one at $62.5 \mathrm{mg}$ b.i.d. at the time of the pharmacokinetic substudy. Pharmacokinetic variables were normalised to a dose of $125 \mathrm{mg}$ b.i.d. This is justified in view of the small dose range investigated in this study and because the pharmacokinetics of bosentan are proportional to dose over a wide dose range [27].

\section{Analysis}

Results are expressed as mean \pm SD or as median. Baseline and post-baseline values for $6 \mathrm{MWD}$ and haemodynamic variables were compared using a two-sided paired t-test. For the subgroup of patients for whom baseline data, data after 4 months and data at last evaluation were available, comparisons were made using ANOVA. Changes in NYHA FC were compared using the Chi-squared test. A p-value $<0.05$ was to be considered statistically significant. Analyses of event-free status were performed using an intention-to-treat approach and the Kaplan-Meier method. The date of initiation of bosentan therapy was the starting point for determining the follow-up duration and estimating survival. Patients lost to follow-up were censored as of the date of the last bosentan prescription.

\section{RESULTS}

Between December 2002 and July 2009, 77 patients with newly diagnosed PoPH were evaluated in our centre. Among them, 10 did not receive any PAH-specific therapy, 17 were treated with first-line sildenafil, 11 with i.v. epoprostenol, one with inhaled iloprost and four with combination therapy (bosentan in association with sildenafil or epoprostenol). Finally, 34 patients were given first-line bosentan monotherapy and constituted the patient population of this study. Patient demographics at baseline, as well as aetiologies of liver disease, are shown in table 1 . Six patients had portal thrombosis without cirrhosis. Among the 28 patients with cirrhosis, 19 were classified into C-P class A and nine into class B. Five patients with C-P class B cirrhosis were included in the pharmacokinetic substudy. No patient displayed positive acute vasodilator response to inhaled nitric oxide at first haemodynamic assessment.

\section{Short-term efficacy}

Short-term evaluation was performed after mean \pm SD $5 \pm 2$ months after bosentan initiation. Significant improvements from baseline were observed in 6MWD, NYHA FC and haemodynamic variables (table 2). Pulmonary haemodynamic data significantly improved with an increase in cardiac index and a decrease in PVR by a mean of $39 \%$ and $31 \%$, respectively.

The short-term haemodynamic response was significantly better in patients with C-P class B cirrhosis compared with those with C-P class A cirrhosis or with noncirrhotic portal hypertension (fig. 1). Individual data for patients with C-P class B cirrhosis after first evaluation of treatment are shown in table 3. Notably, PVRs were either near-normalised or normalised ( $<3$ Wood units) in a subset of patients with cirrhosis C-P class B after 4 months of bosentan treatment.

TABLE 1 Baseline demographic, clinical, biological and
haemodynamic characteristics

Data are presented as $\mathrm{n}$ or mean \pm SD. NYHA FC: New York Heart Association functional class; 6MWD: 6-min walk distance; Pra: right atrial pressure; Ppa: pulmonary artery pressure; $P$ pcw: pulmonary capillary wedge pressure; PVR: pulmonary vascular resistance; $\mathrm{Sv}_{1} \mathrm{O}_{2}$ : mixed venous oxygen saturation; AST: aspartate aminotransferase; ALT: alanine aminotransferase.

After first evaluation, bosentan monotherapy was continued in 27 patients and sildenafil was added in seven. All these seven patients had cirrhosis C-P class A.

\section{Long-term efficacy}

24 patients were assessed at least three times under bosentan monotherapy. The last evaluation of these patients was conducted $35 \pm 16$ months (range 12-75 months) after bosentan initiation. The remaining 10 patients did not undergo longterm evaluation on monotherapy with bosentan for the following reasons: seven patients received sildenafil in addition to bosentan after the first evaluation, sildenafil was introduced to replace bosentan in one patient because of liver toxicity and two patients were receiving bosentan monotherapy but had only one evaluation at cut-off. In patients who were evaluated at least three times, improvements in haemodynamic variables observed in the short term were largely maintained, with significant improvement in NYHA FC and increase over baseline in cardiac index and PVR (table 2). 6MWD significantly improved from baseline in this group of patients at final assessment. Individual data for patients with C-P class B after $37 \pm 25$ months of treatment are shown in table 3. 
TABLE 2 Functional class, exercise capacity and haemodynamics at baseline and on bosentan monotherapy and follow-up evaluations in patients treated exclusively by bosentan monotherapy

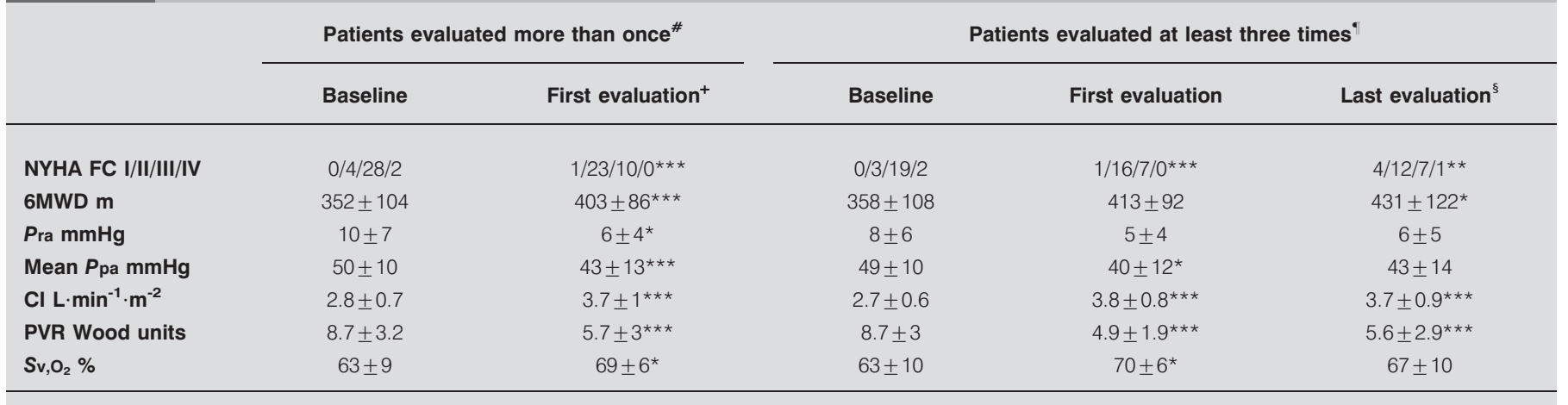

Data are presented as $\mathrm{n}$ or mean $\pm \mathrm{SD}$. NYHA FC: New York Heart Association functional class; 6MWD: 6-min walk distance; Pra: right atrial pressure; Ppa: pulmonary artery pressure; $\mathrm{Cl}$ : cardiac index; PVR: pulmonary vascular resistance; $\mathrm{Sv}_{\mathrm{,}} \mathrm{O}_{2}:$ mixed venous oxygen saturation. ${ }^{*}: \mathrm{n}=34 ;{ }^{~}{ }^{*}: \mathrm{n}=24{ }^{+}{ }^{+}$: first evaluation performed on bosentan monotherapy $5 \pm 2$ months after treatment initiation (range 4-12 months); ${ }^{\text {s: }}$ last evaluation performed on bosentan monotherapy $35 \pm 16$ months after treatment initiation (range 12-75 months). *: $p<0.05$ versus baseline; ${ }^{* *}: p<0.01$ versus baseline; ${ }^{* *}: p<0.001$ versus baseline.

\section{Effect of bosentan therapy on overall and event-free survival} At the cut-off date (May 31, 2010), the mean follow-up period was $43 \pm 19$ months. Event-free survival estimates were $82 \%$, $63 \%$ and $47 \%$ at 1,2 and 3 yrs, respectively. Event-free survival curves were similar in patients with noncirrhotic portal hypertension or C-P class A cirrhosis and patients with more severe C-P class B cirrhosis (fig. 2a).

12 patients required additional $\mathrm{PAH}$-specific therapies during the follow-up period. All of them received sildenafil in combination with bosentan.

Four patients died during follow-up at 21, 36, 61 and 67 months, respectively. Three had cirrhosis C-P class A and 1 had cirrhosis C-P class B. The cause of death was right heart failure for three patients and hepatocellular carcinoma for one patient (fig. 2b).

\section{Safety}

A significant elevation of liver enzymes ( $>3 \times$ the ULN) was observed in seven patients, corresponding to an annual rate of
5.5\%. Among these seven patients, bosentan was discontinued in three patients with C-P class A cirrhosis after 6, 15 and 33 months, and two others with C-P class B cirrhosis after 16 and 58 months due to increased liver enzymes. Bosentan was discontinued in three patients who received moderately high dosage (62.5 $\mathrm{mg}$ b.i.d.) because of a moderately elevated level of liver enzymes at baseline of $1-3 \times$ the ULN. In all cases, sildenafil was introduced to replace bosentan. Annual rate of increase in liver enzymes was $4.1 \%$ (95\% CI $0.2-8 \%$ ) in patients with noncirrhotic portal hypertension and with cirrhosis C-P class A, and $12.4 \%$ (95\% CI 0.7-25.6\%) in patients with cirrhosis C-P class B (not statistically significant). In six cases, normalisation of hepatic transaminase levels was observed during the 3 months following bosentan dose reduction or discontinuation. In one other case, persistently abnormal liver enzyme levels were attributed to underlying hepatocellular carcinoma.

\section{Pharmacokinetics}

Figure 3 shows the dose-normalised plasma concentrationtime profiles of bosentan in five $\mathrm{PoPH}$ patients with C-P class B
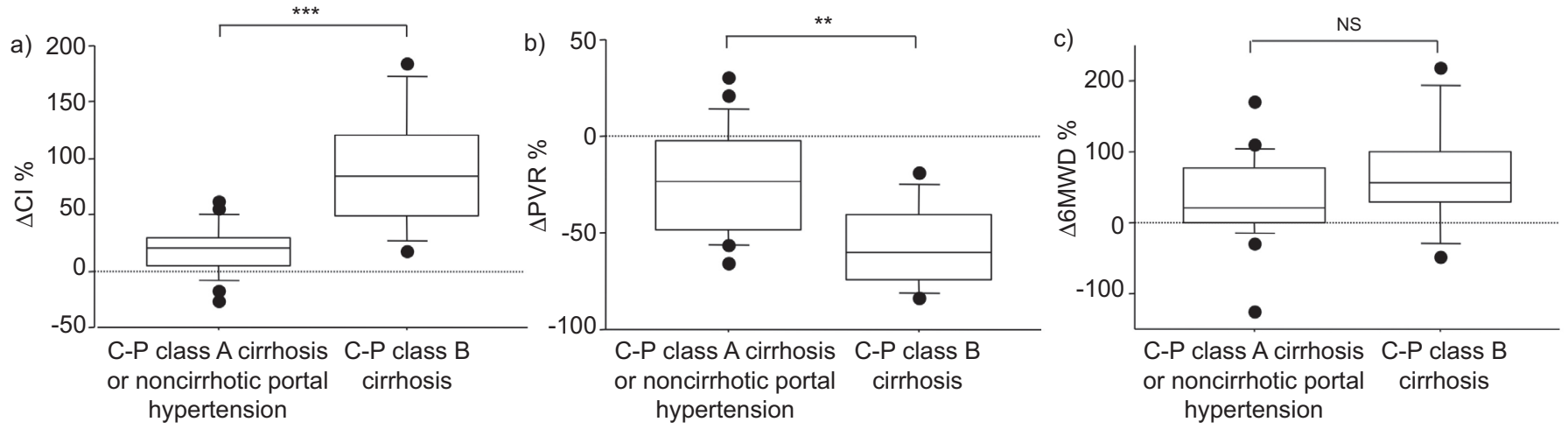

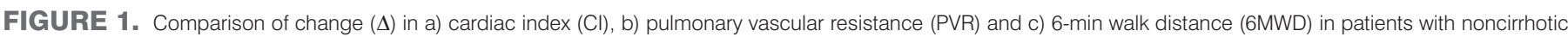

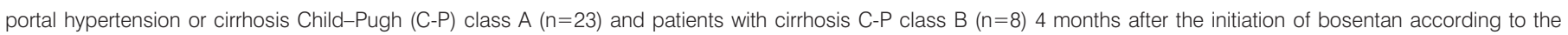

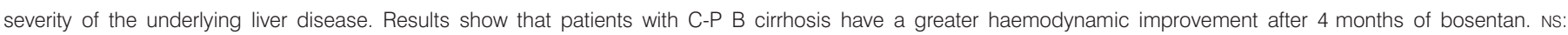
nonsignificant. ${ }^{\star *}: \mathrm{p}<0.01 ; * \star \star *: p<0.001$ 


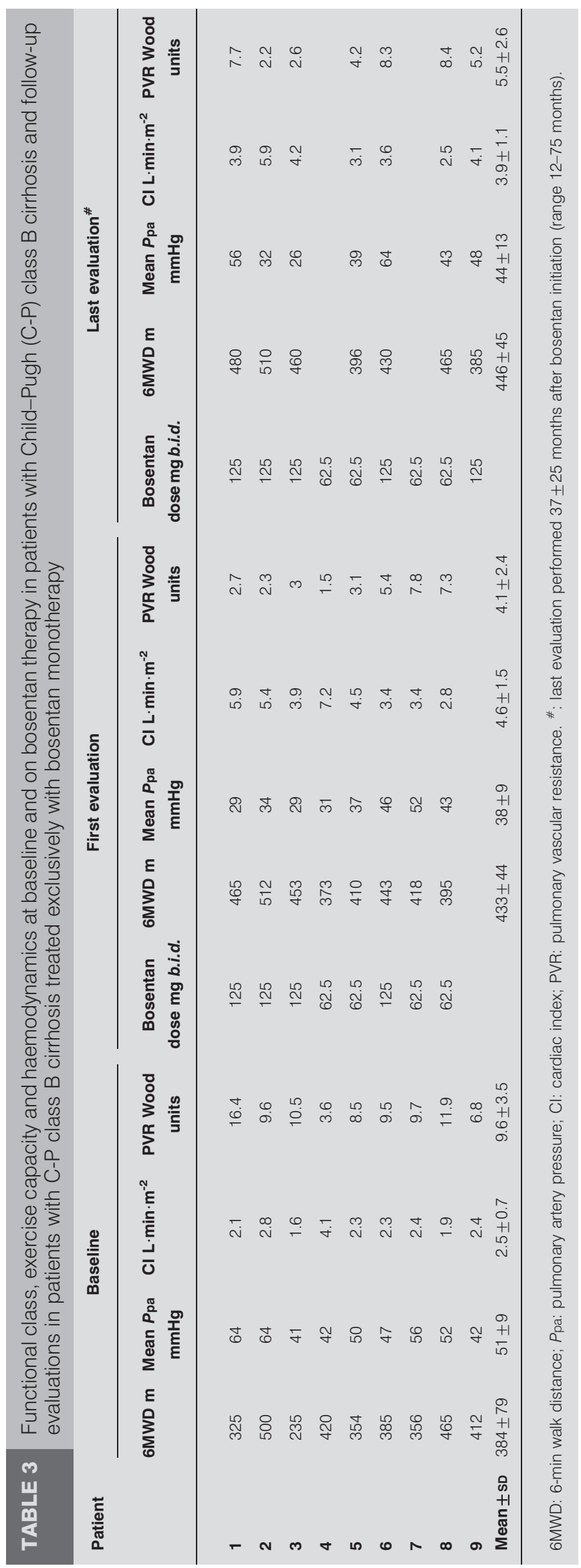

a)

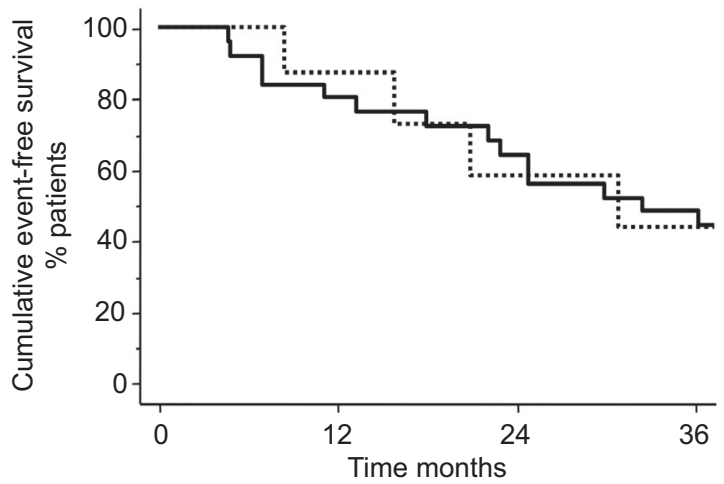

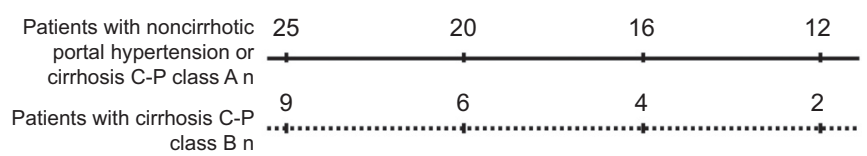

b)



FIGURE 2. Kaplan-Meier estimates of a) event-free and b) cumulative survival in 34 patients with portopulmonary hypertension treated with first-line bosentan therapy according to the severity of the underlying liver disease. C-P: Child-Pugh.

cirrhosis and three patients with IPAH. The profiles of the three bosentan metabolites measured showed a similar course and difference between the groups (data not shown). The pharmacokinetic variables derived from the individual plasma concentration-time profiles are presented in table 4 .

\section{DISCUSSION}

There are currently few data on the use of PAH-specific therapies in $\mathrm{PoPH}$, particularly in patients with more advanced hepatic disease. This study was one of the largest to date in this patient population and the first to include a significant number of patients in C-P class B. Treatment with bosentan was associated with short-term improvements in functional status, 6MWD and haemodynamics that were maintained over the long term. In patients with more advanced liver disease C-P class B, haemodynamic response was greater leading to a normalisation or near-normalisation of PVR in some patients. Moreover, long-term treatment with bosentan was well tolerated in patients with mild or more severe cirrhosis. 


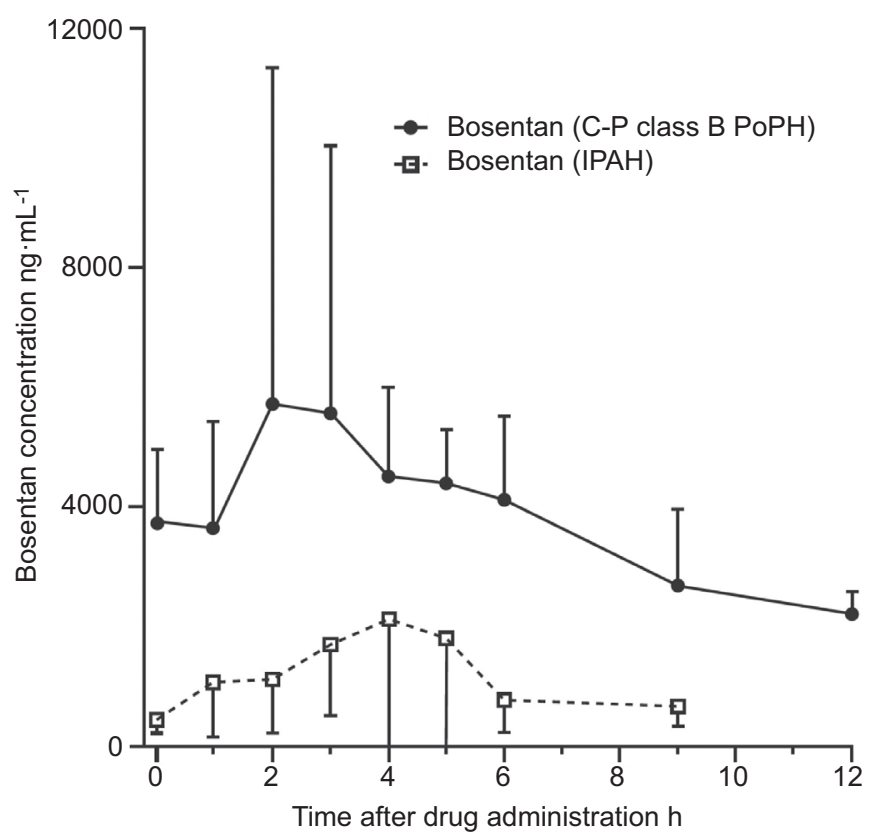

FIGURE 3. Mean $\pm S D$ dose-normalised plasma concentration-time profiles of bosentan in portopulmonary hypertension ( $\mathrm{PoPH}$ ) patients with Child-Pugh (C-P) class $B$ cirrhosis $(n=5)$ or patients with idiopathic pulmonary arterial hypertension (IPAH) without comorbid portal hypertension $(n=3)$. Concentrations from patients treated with doses of $62.5 \mathrm{mg}$ b.i.d. were normalised to a dose of $125 \mathrm{mg}$.

The mean treatment duration of $47 \pm 20$ months enables an adequate evaluation of the potential long-term benefits of bosentan therapy in these patients. Significant improvement in haemodynamic variables between baseline and follow-up evaluations is encouraging, as it is correlated with a better prognosis [7]. Indeed, patients with a lower cardiac index are usually at higher risk of death [7]. Therefore, improvement in cardiac index in this study suggests that bosentan may be beneficial and improve survival. In these patients with portal hypertension, bosentan seems at least as effective as previously observed in double-blind, placebo-controlled studies in patients with IPAH and PAH associated with connective tissue disease [28-30].
The pronounced improvements observed in patients with liver disease in C-P class B are of particular interest. This is an important result, because risk of mortality is higher for $\mathrm{PoPH}$ patients with more severe liver disease [7]. Despite the existence of a more severe liver dysfunction in patients with cirrhosis C-P class B, which is known to be a poorer prognostic factor, the event-free survival is equivalent to patients with either mild cirrhosis or noncirrhotic portal hypertension and patients with more severe cirrhosis. Normalisation or nearnormalisation of haemodynamics is exceptional with PAHspecific therapy in patients with either IPAH or PAH associated with concomitant disease. This type of response is generally observed in patients with $\mathrm{PAH}$ associated with inflammatory conditions, such as HIV infection or connective tissue diseases [31, 32]. Inflammatory processes are more important in patients with more advanced hepatic disorders, which could explain pronounced improvement in these patients. In patients with portal hypertension, the development of portosystemic shunts, a decrease in liver phagocytic capacity and increased frequency of bacterial translocation facilitate the circulation of pulmonary cytokines, pro-angiogenic factors or bacterial endotoxins, which probably induce pulmonary vascular endothelium dysfunction and increase production of endothelin. Data from this study also suggest a role of endothelin in the pathological process of PoPH. In addition, there is evidence to suggest that endothelin- 1 may be aetiologically relevant in portal hypertension and hepatic fibrotic remodelling [33-36].

Another hypothesis explaining differences of response in patients with more severe cirrhosis could be increased systemic exposure to bosentan and/or its active metabolite due to impaired metabolism in such patients. Results from the pharmacokinetic substudy presented here suggest that this is indeed the case, as the maximal concentration of bosentan was considerably higher in $\mathrm{PoPH}$ patients with cirrhosis C-P class B than in those with IPAH, even though patients with cirrhosis were taking lower doses of bosentan at the time of the pharmacokinetic substudy. The underlying mechanism for this difference is probably a decrease in the efficiency of organic anion transporter peptide, the transporter responsible for uptake of bosentan into hepatocytes, rather than enzymes responsible for bosentan metabolism in the liver (cytochrome

TABLE 4 Pharmacokinetic variables of bosentan and its metabolites in patients with portopulmonary hypertension $(\mathrm{PoPH} ; \mathrm{n}=5)$ and idiopathic pulmonary arterial hypertension (IPAH; $\mathrm{n}=3$ )

\begin{tabular}{|c|c|c|c|c|c|c|}
\hline & \multicolumn{2}{|c|}{$t \max \mathrm{h}$} & \multicolumn{2}{|c|}{$C_{\max } \mathrm{ng} \cdot \mathrm{mL}^{-1}$} & \multicolumn{2}{|c|}{$A U C_{\tau} \mathbf{n g} \cdot \mathrm{mL}^{-1} \cdot \mathbf{h}^{-1}$} \\
\hline & PoPH & IPAH & PoPH & IPAH & PoPH & IPAH \\
\hline Bosentan & $4(2-6)$ & $4(3-4)$ & $53.3(22.4-127)$ & $14.6(1.27-168)$ & 360 (212-613) & 76.1 (9.07-638) \\
\hline Ro 47-8634 & $6(5-6)$ & $3(3-4)$ & $0.753(0.393-1.45)$ & $0.351(0.0439-2.81)$ & $6.39(3.21-12.7)$ & $2.40(0.427-13.5)$ \\
\hline Ro $48-5033$ & $2(0-9)$ & $3(0-5)$ & $13.2(7.98-21.8)$ & $1.46(0.158-13.5)$ & $106(58.4-192)$ & $8.57(1.28-57.2)$ \\
\hline
\end{tabular}

Data are presented as geometric mean (median) for $t$ max and geometric mean (95\% confidence interval) for $\mathrm{Cmax}$ and area under the curve for one dosing interval (AUC $\tau$ )




P450 isoenzymes), because the extent of the increase in exposure was similar for bosentan and its metabolites. Although only three patients with IPAH were included as comparators in this study, the pharmacokinetic results obtained were very similar to those obtained in a larger group of PAH patients [27]. More detailed pharmacokinetic studies are warranted to investigate the influence of more severe forms of liver cirrhosis on the disposition of ERAs.

The use of ERAs is associated with an increased frequency of elevated aminotransferase levels, and monthly liver function tests are required for all patients treated with them. Therefore, potential concerns exist regarding their safety in patients with either cirrhosis or impaired liver function, such as those analysed in this study. Liver damage could also change the metabolism of bosentan in such patients. However, the results presented here suggest that there is no difference between patients with PoPH and those with other forms of PAH. The annual rate of liver enzyme elevations observed in this study is similar to that previously reported in the post-marketing surveillance of bosentan [37] and in other trials in PAH [28-30]. They usually develop gradually, remain asymptomatic, and are generally reversible either spontaneously or after dose reduction or discontinuation. We observed a trend for a higher annual rate of aminotransferases elevation and higher incidence of bosentan discontinuation in patients with more severe cirrhosis. In all cases, liver disturbance was reversible and without impact on liver disease evolution. However, close monitoring of liver enzymes should be conducted in patients with more severe cirrhosis C-P class B. Despite a good haemodynamic response observed in patients with advanced liver disease, bosentan should probably be used in these patients both with caution and after having considered the use of alternative treatments without liver liability as first-line therapy. In addition, the results of the pharmacokinetic substudy and responses to treatment observed in patients with more advanced liver disease may suggest that the use of bosentan at a dose of $62.5 \mathrm{mg}$ b.i.d. may be sufficient.

A recently published observational study has reported on the effects of ambrisentan, a selective antagonist of the ETA receptor subtype, in 13 patients with moderate-to-severe PoPH associated with mild cirrhosis (C-P class A). Treatment was associated with a significant reduction in mean $P$ pa and PVR without adverse effects on liver function tests [38]. This drug, which is considered to have a minimal effect on liver function, could also be an interesting option in patients with more severe cirrhosis. This option must be properly evaluated.

The main limitation of our study is its retrospective open-label design. Accordingly, data presented here should be interpreted with caution. Another limitation is the potential selection bias in the long-term data analysed in this study; it is possible that the patient population was enriched for patients who responded to treatment. This is especially true for the long-term analysis of 6MWD. Because of the small numbers involved and the variability of dosing, the pharmacokinetic data also must be interpreted with caution. It is unfortunate that plasma bosentan levels were not measured in all patients, especially as samples were obtained only from three control patients. However, the pharmacokinetic properties of bosentan in these control patients were in close accordance with those assessed in a larger historical control group [27]. Furthermore, the fact that bosentan plasma levels were not measured, at least in some C-P class A patients, is also a limitation of the study. The pharmacokinetics of bosentan in C-P class A patients have been studied previously [21], but these patients were not diagnosed with PoPH. Finally, plasma levels may not necessary reflect tissular drug action on the receptor. Nevertheless, this study represents a considerable advance on previously available data and provides a rationale for further studies investigating this important patient population. It is unlikely that any future studies will be placebo controlled, given the poor prognosis of patients with PAH who do not receive treatment [39]. A prospective open-label trial would provide valuable data. Furthermore, pharmacological data should be enriched by a larger study comparing the pharmacokinetics of bosentan between patients with mild cirrhosis and more severe cirrhosis.

These data confirm the benefit of bosentan in the treatment of patients with $\mathrm{PoPH}$, especially with regards to haemodynamic improvements. In particular, this study suggests that the greatest positive haemodynamic responses are in patients with C-P class B cirrhosis. The safety profile of bosentan in patients with PoPH was generally consistent with previous studies including PAH patients without cirrhosis. However, special care must be taken with patients with more severe cirrhosis due to the potential liver toxicity of this treatment.

\section{STATEMENT OF INTEREST}

Statements of interest for L. Savale, X. Jaïs, D. Montani, M. Humbert, J. Dingemanse, G. Simonneau and O. Sitbon can be found at www.erj. ersjournals.com/site/misc/statements.xhtml

\section{REFERENCES}

1 Simonneau G, Robbins IM, Beghetti M, et al. Updated clinical classification of pulmonary hypertension. J Am Coll Cardiol 2009; 54: Suppl. 1, S43-S54.

2 Hadengue A, Benhayoun MK, Lebrec D, et al. Pulmonary hypertension complicating portal hypertension: prevalence and relation to splanchnic hemodynamics. Gastroenterology 1991; 100: 520-528.

3 Colle IO, Moreau R, Godinho E, et al. Diagnosis of portopulmonary hypertension in candidates for liver transplantation: a prospective study. Hepatology 2003; 37: 401-409.

4 Hoeper MM, Krowka MJ, Strassburg CP. Portopulmonary hypertension and hepatopulmonary syndrome. Lancet 2004; 363: 1461-1468.

5 Halank M, Ewert R, Seyfarth HJ, et al. Portopulmonary hypertension. J Gastroenterol 2006; 41: 837-847.

6 Humbert M, Sitbon O, Chaouat A, et al. Pulmonary arterial hypertension in France: results from a national registry. Am J Respir Crit Care Med 2006; 173: 1023-1030.

7 Le Pavec J, Souza R, Herve P, et al. Portopulmonary hypertension: survival and prognostic factors. Am J Respir Crit Care Med 2008; 178: 637-643.

8 Robalino BD. Association between primary pulmonary hypertension and portal hypertension: analysis of its pathophysiology and clinical, laboratory and hemodynamic manifestations. J Am Coll Cardiol 1991; 17: 492-498.

9 Kawut SM, Taichman DB, Ahya VN, et al. Hemodynamics and survival of patients with portopulmonary hypertension. Liver Transpl 2005; 11: 1107-1111. 
10 Krowka MJ, Miller DP, Barst RJ, et al. Portopulmonary hypertension: a report from the US-based REVEAL Registry. Chest 2012; 141: 906-915.

11 Benza RL, Miller DP, Gomberg-Maitland M, et al. Predicting survival in pulmonary arterial hypertension: insights from the Registry to Evaluate Early and Long-Term Pulmonary Arterial Hypertension Disease Management (REVEAL). Circulation 2010; 122: 164-172.

12 Halank M, Miehlke S, Hoeffken G, et al. Use of oral endothelinreceptor antagonist bosentan in the treatment of portopulmonary hypertension. Transplantation 2004; 77: 1775-1776.

13 Grander W, Eller P, Fuschelberger R, et al. Bosentan treatment of portopulmonary hypertension related to liver cirrhosis owing to hepatitis C. Eur J Clin Invest 2006; 36: Suppl. 3, 67-70.

14 Hinterhuber L, Graziadei IW, Kahler CM, et al. Endothelinreceptor antagonist treatment of portopulmonary hypertension. Clin Gastroenterol Hepatol 2004; 2: 1039-1042.

15 Neuhofer W, Gulberg V, Gerbes AL. Endothelin and endothelin receptor antagonism in portopulmonary hypertension. Eur J Clin Invest 2006; 36: Suppl. 3, 54-61.

16 Stahler G, von Hunnius P. Successful treatment of portopulmonary hypertension with bosentan: case report. Eur J Clin Invest 2006; 36: Suppl. 3, 62-66.

17 Barth F, Gerber PJ, Reichen J, et al. Efficiency and safety of bosentan in child $\mathrm{C}$ cirrhosis with portopulmonary hypertension and renal insufficiency. Eur J Gastroenterol Hepatol 2006; 18: 1117-1119.

18 Kuntzen C, Gulberg V, Gerbes AL. Use of a mixed endothelin receptor antagonist in portopulmonary hypertension: a safe and effective therapy? Gastroenterology 2005; 128: 164-168.

19 Hoeper MM, Seyfarth HJ, Hoeffken G, et al. Experience with inhaled iloprost and bosentan in portopulmonary hypertension. Eur Respir J 2007; 30: 1096-1102.

20 Hoeper MM, Halank M, Marx C, et al. Bosentan therapy for portopulmonary hypertension. Eur Respir J 2005; 25: 502-508.

21 van Giersbergen PL, Popescu G, Bodin F, et al. Influence of mild liver impairment on the pharmacokinetics and metabolism of bosentan., a dual endothelin receptor antagonist. J Clin Pharmacol 2003; 43: 15-22.

22 Rodriguez-Roisin R, Krowka MJ, Herve P, et al. Pulmonaryhepatic vascular disorders (PHD). Eur Respir J 2004; 24: 861-880.

23 Sitbon $\mathrm{O}$, Humbert $\mathrm{M}$, Jais $\mathrm{X}$, et al. Long-term response to calcium channel blockers in idiopathic pulmonary arterial hypertension. Circulation 2005; 111: 3105-3111.

24 Galie N, Hoeper MM, Humbert M, et al. Guidelines for the diagnosis and treatment of pulmonary hypertension: the Task Force for the Diagnosis and Treatment of Pulmonary Hypertension of the European Society of Cardiology (ESC) and the European Respiratory Society (ERS), endorsed by the
International Society of Heart and Lung Transplantation (ISHLT). Eur Respir J 2009; 34: 1219-1263.

25 Cohen $\mathrm{H}$, Chahine $\mathrm{C}$, Hui A, et al. Bosentan therapy for pulmonary arterial hypertension. Am J Health Syst Pharm 2004; 61: 1107-1119.

26 Dell D, Lausecker B, Hopfgartner G, et al. Evolving bioanalytical methods for the cardiovascular drug bosentan. Chromatographia 2002; 55: S115-S119.

27 Dingemanse J, van Giersbergen PL. Clinical pharmacology of bosentan, a dual endothelin receptor antagonist. Clin Pharmacokinet 2004; 43: 1089-1115.

28 Channick R, Badesch DB, Tapson VF, et al. Effects of the dual endothelin receptor antagonist bosentan in patients with pulmonary hypertension: a placebo-controlled study. J Heart Lung Transplant 2001; 20: 262-263.

29 Rubin LJ, Badesch DB, Barst RJ, et al. Bosentan therapy for pulmonary arterial hypertension. N Engl J Med 2002; 346: 896-903.

30 Galie N, Rubin L, Hoeper M, et al. Treatment of patients with mildly symptomatic pulmonary arterial hypertension with bosen$\tan$ (EARLY study): a double-blind, randomised controlled trial. Lancet 2008; 371: 2093-2100.

31 Degano B, Yaici A, Le Pavec J, et al. Long-term effects of bosentan in patients with HIV-associated pulmonary arterial hypertension. Eur Respir J 2009; 33: 92-98.

32 Jais $\mathrm{X}$, Launay D, Yaici A, et al. Immunosuppressive therapy in lupus- and mixed connective tissue disease-associated pulmonary arterial hypertension: a retrospective analysis of twenty-three cases. Arthritis Rheum 2008; 58: 521-531.

33 Benjaminov FS, Prentice M, Sniderman KW, et al. Portopulmonary hypertension in decompensated cirrhosis with refractory ascites. Gut 2003; 52: 1355-1362.

34 Chan CC, Wang SS, Lee FY, et al. Endothelin-1 induces vasoconstriction on portal-systemic collaterals of portal hypertensive rats. Hepatology 2001; 33: 816-820.

35 Kojima H, Sakurai S, Kuriyama S, et al. Endothelin-1 plays a major role in portal hypertension of biliary cirrhotic rats through endothelin receptor subtype B together with subtype A in vivo. J Hepatol 2001; 34: 805-811.

36 Tieche S, De Gottardi A, Kappeler A, et al. Overexpression of endothelin-1 in bile duct ligated rats: correlation with activation of hepatic stellate cells and portal pressure. J Hepatol 2001; 34: 38-45.

37 Humbert M, Segal ES, Kiely DG, et al. Results of European postmarketing surveillance of bosentan in pulmonary hypertension. Eur Respir J 2007; 30: 338-344.

38 Cartin-Ceba R, Swanson K, Iyer V, et al. Safety and efficacy of ambrisentan for the treatment of portopulmonary hypertension. Chest 2011; 139: 109-114.

39 D'Alonzo GE, Barst RJ, Ayres SM, et al. Survival in patients with primary pulmonary hypertension: results from a national prospective study. Ann Intern Med 1991; 115: 343-349. 